\title{
ARE CYTOCHROME $B$ GENE SEQUENCING AND POLYMORPHISM-SPECIFIC POLYMERASE CHAIN REACTION AS RELIABLE AS MULTILOCUS ENZYME ELECTROPHORESIS FOR IDENTIFYING LEISHMANIA SPP. FROM ARGENTINA?
}

\author{
JORGE D. MARCO, * HIROSHI UEZATO, TATSUYUKI MIMORI, PAOLA A. BARROSO, MASATAKA KORENAGA, \\ SHIGEO NONAKA, MIGUEL A. BASOMBRÍO, NÉSTOR J. TARANTO, AND YOSHIHISA HASHIGUCHI \\ Department of Parasitology, Kochi Medical School, Kochi University, Kochi, Japan; Instituto de Patología Experimental, Facultad de \\ Ciencias de la Salud, Universidad Nacional de Salta / Consejo Nacional de Investigaciones Científicas y Técnicas, Salta, Argentina; \\ Department of Dermatology, Faculty of Medicine, University of the Ryukyus, Okinawa, Japan; Department of Tumor Genetics \& \\ Biology, Graduate School of Medical Science, Kumamoto University, Kumamoto, Japan; Instituto de Enfermedades Tropicales, Sede \\ Regional Orán, Universidad Nacional de Salta, San Ramón de la Nueva Orán, Argentina
}

\begin{abstract}
Recently, two techniques, polymerase chain reaction (PCR) amplification and sequencing of cytochrome $b$ gene (cyt b gene sequencing) and polymorphism-specific PCR (PS-PCR) were recommended for Leishmania species identification. Before this study, however, the accuracy of these methods had not been tested against the multilocus enzyme electrophoresis, the current gold standard technique on this task. Therefore, a trial was done for the first time to compare the results obtained by these techniques, using 17 Argentinean Leishmania stocks in independent assays. For all the stocks examined, the same results at species level were obtained by the three techniques. Among them, 14 were assigned to L. (Viannia) braziliensis, and three to L. (V.) guyanensis. The two techniques, cyt b gene sequencing and PS-PCR, were able to distinguish between all the proven species responsible for leishmaniases in Argentina. Thus, both techniques were validated and could be used independently for the species designation of Leishmania parasites in the country.
\end{abstract}

\section{INTRODUCTION}

The leishmaniases are parasitic diseases that threaten 350 million people in 88 countries on 4 continents, with an annual incidence of new cases estimated between 1.5 and 2 million. They are caused by protozoan flagellates that belong to Leishmania genus, which includes around 30 taxa. ${ }^{1}$ There is no direct correlation between these Leishmania species and the specific clinical patterns induced except several cases, ${ }^{2}$ and they have shown different responses to the chemotherapy. ${ }^{3}$ In addition, it is common for different species to coexist in the same endemic areas, as seen in Argentina, where Leishmania (Viannia) braziliensis, L. (V.) guyanensis, and L. (Leishmania) amazonensis have been incriminated as the causative agents of human leishmaniases by molecular methods to date. ${ }^{4-9}$ In particular, substantial evidence exists that the diseases caused by L. (V.) braziliensis and L. (V.) guyanensis differ in their clinical presentation, diagnostic approach required, and therapeutic response to antimonials. ${ }^{10,11}$

For these reasons, the Leishmania spp. discrimination is important not only from an epidemiologic perspective but also from the clinical ones to improve the patients' prognosis, diagnostic methods, and to monitor clinical outcomes. ${ }^{12,13}$ Moreover, the accurate identification of these parasites must be based on molecular approaches because parasitological, clinical, or epidemiologic features are by themselves insufficient for this task. ${ }^{14}$

Among the molecular methods applied on the Leishmania spp. identification, the DNA-based techniques have been used increasingly. One of them, the polymerase chain reaction (PCR) amplification and sequencing of cytochrome $b$ gene method ( $c y t$ b gene sequencing) has recently been established as a useful tool for the identification and phyloge-

\footnotetext{
* Address correspondence to Jorge D. Marco, Department of Parasitology, Kochi Medical School, Kochi University, Kohasu, Oko, Nankoku, Kochi 783-8505, Japan. E-mail: marcojd@med.kochims.ac.jp
}

netic studies of the Leishmania genus, being able to differentiate among the species and from other trypanosomatids. ${ }^{15}$ Another of these techniques, the polymorphism-specific PCR (PS-PCR), has been used for the Leishmania spp. identification based on the presence of bands amplified for subgenus or species-specific primers, without requiring other procedures. ${ }^{16}$

Since both $c y t \mathrm{~b}$ gene sequencing and PS-PCR, as with the other DNA-based techniques, have some advantages over the classically used techniques on the Leishmania spp. identification, such as isoenzymes, serodemes, or schizodeme analysis, ${ }^{12}$ they may have an advantage of the application in field work, particularly in Argentina. However, to be used widely as reliable and effective methods, it is necessary to evaluate their efficacy in restricted endemic areas. ${ }^{17}$

This article reports for the first time a double-blind assay designed to test the accuracy of the $c y t$ b gene sequencing and PS-PCR methods on Leishmania spp. identification, compared with multilocus enzyme electrophoresis (MLEE), the current gold standard technique, ${ }^{1}$ using a panel of Argentinean Leishmania isolates, as a required step in their optimization. The results are discussed in terms of the probable applications of these techniques, highlighting their advantages, on the areas of diagnosis, clinico-pathology, and epidemiology of leishmaniases in Argentina.

\section{MATERIALS AND METHODS}

Parasites. Seventeen Leishmania stocks, characterized by MLEE analyzing 12 loci, were included in this study. Sixteen of them were isolated in the northern areas of Argentina, one from a canine and 15 from humans, all American tegumentary leishmaniasis (ATL) cases. Their enzymatic profiles, phylogenetic relationships, and clinico-epidemiologic characteristics were reported previously. ${ }^{4}$ The remaining isolate, named MHOM/AR/99/DMZ, was obtained from the stock MHOM/AR/99/JDM1 after performing culture-mouse passages 4 times, as follows: JDM1 promastigotes were cultured for $10 \mathrm{ds}$ in $10 \mathrm{~mL}$ of sterile liver infusion tryptose medium 
supplemented with $100 \mathrm{U} / \mathrm{mL}$ Penicillin and $50 \mu \mathrm{g} / \mathrm{mL}$ Streptomycin (PE), 20\% heat inactivated fetal bovine serum (hiFBS). ${ }^{18}$ They were harvested and $0.05 \mathrm{~mL}$ of a suspension at $20.10^{7}$ promastigotes $/ \mathrm{mL}$ was inoculated into right footpads of 4-week-old mice. After 3 weeks of inoculation, the footpad tissue was aseptically removed, homogenized, and cultured again in the same medium. This procedure was repeated 4 times.

Five World Health Organization (WHO) reference strains: L. (V.) braziliensis, HOM/BR/75/M2904; L. (V.) panamensis, MHOM/PA/71/LS94; L. (V.) guyanensis, MHOM/BR/75/ M4147; L. (L.) amazonensis, MHOM/BR/73/M2269; L. (L.) mexicana, MNYC/BZ/62/M379; and one L. (V.) braziliensis Ecuadorian strain: $\mathrm{MHOM} / \mathrm{EC} / 88 / \mathrm{INH}-03$ were used as controls.

Samples preparation for DNA analysis. Promastigotes of each isolate were cultured in RPMI 1640 medium supplemented with $\mathrm{PE}$ and $10 \%$ of hiFBS, diluting gradually to a final volume of $8 \mathrm{~mL}$ for $7 \mathrm{ds}$. Two tubes for each isolate were done. After washing 4 times (2,500 rpm, 10 minutes) with sterile PBS, $2 \mathrm{~mL}$ of TE buffer $(10 \mathrm{mM}$ Tris- $\mathrm{HCl}, \mathrm{pH}=8$, $1 \mathrm{mM}$ EDTA) or $99 \%$ ethanol were added to the pellet of each tube. These samples were aliquoted and stored at $-20^{\circ} \mathrm{C}$ until use. This procedure was carried out at the same time that the pellets of promastigotes for MLEE were prepared.

Detection of cytochrome $b$ gene using polymerase chain reaction. To extract the genomic DNA from each of the samples prepared, a genomic extraction kit (i.e., Genomic Prep Cell and Tissue DNA Isolation Kit (AmershamBiosciences, USA)) was used, following the protocol and methods described by the company. The PCR was performed with Ex-Taq polymerase (Takara, Japan) under the following conditions: initial denaturation at $94^{\circ} \mathrm{C}$ for 3 minutes followed by 35 cycles of $94^{\circ} \mathrm{C}$ for 1 minute, $50^{\circ} \mathrm{C}$ for 1 minute, and $72^{\circ} \mathrm{C}$ for 1 minute. Two hundred ng of parasite DNA as a template, LCBF1 forward primer (5'-GGTGTAGGTTTTAGTYTAGG-3'), and LCBR2 reverse primer (5'-CTACAATAAACAAATCATAATATRCAATT-3') were used for the $c y t$ b gene amplification. The PCR products were visualized on a $1 \%$ agarose gel.

Cytochrome $\boldsymbol{b}$ gene direct sequencing procedures. The cyt $\mathrm{b}$ gene sequences for each sample were determined following the procedure described previously. ${ }^{15,19}$ Briefly, the amplified fragments, obtained as was described previously, were electrophoresed on 1\% Seakam GTG agarose gel (FMC, USA), excised, and then purified by using the QIA quick Gel Extraction kit (Qiagen, USA). The purified DNA was examined by direct sequencing with the Big Dye Terminator Cycle Sequencing kit (PE Biosystems, USA) and ABI PRISM ${ }^{\mathrm{TM}} 310$ Genetic Analyzer. The sequencing primers used were LCBF1, LCBR2, LCYT B F4L (5'-TGTTATTGAATATGAGGTAGTG-3') and LCYT B R4 (5'-GAACTCATAAAATAATGTAAACAAAA-3'). The obtained sequences, assembled and edited by Genetyx Mac 11.0.0 (Software Development Co. Ltd, Japan) were compared with the reference strain ones, available from DDBJ/EMBL/ GeneBank nucleotide sequence databases with accession numbers from AB095957 to AB095970.

Polymorphism-specific polymerase chain reaction procedures. The DNA samples obtained from the Leishmania stocks as was described previously were centrifuged at 8000 rpm for 5 minutes and the pellets were treated with Insta-
Gene Matrix (Bio Rad, Hercules, CA). A dilution of 1/100 in MilliQ water of the resulting materials termed "InstaGene DNA preparations", was used as template and subjected to PCR procedures in a GeneAmp PCR System 2400 (PerkinElmer, Wellesley, MA) using Roche GeneAmp XL PCR Kit (Applied Biosystems, Foster City, CA), at a final volume of $15 \mu \mathrm{L}$. For Leishmania subgenus identification, the following conditions were applied: after initial denaturation at $95^{\circ} \mathrm{C}$ for 5 minutes, 35 cycles $\left(30\right.$ seconds at $95^{\circ} \mathrm{C}, 30$ seconds at $60^{\circ} \mathrm{C}$, 30 seconds at $72^{\circ} \mathrm{C}$ ) in the first step. This procedure was carried out using the V1 (5'-GCTTCTCGTTTCGCTTTGAAC$\left.3^{\prime}\right)$ - V2 (5'-CAAGACAAGAAAAAAGGCGGC-3') for the detection of L. (Viannia), and L1 (5'-GGTCACTCGGCATTTTTGC-3')-L2 (5'-GTGCCCTGACTTGCATGTCTA-3') for L. (Leishmania) subgenus.

In the second step, PS-PCR was performed under the following condition: 37 cycles $\left(30\right.$ seconds at $95^{\circ} \mathrm{C}$, annealing for 90 seconds at $70^{\circ} \mathrm{C}$ for the L. (Viannia) group or $60^{\circ} \mathrm{C}$ for the L. (Leishmania) group, and extension for $90 \mathrm{sec}-$ onds at $\left.72^{\circ} \mathrm{C}\right)$, using the primers as follows: b1 $\left(5^{\prime}-\right.$ GTGGGCGTATCTGCTGATGAC-3')-b2 (5' -CAAAAAGCGAGGGACTGCGGA-3') for $L$. (V.) braziliensis, p1 (5'-GGTCGGATCTGCATGCATCAC-3')-p2 (5' CAAAAAGCGAGGGACTGCGGG-3') for $L$. (V.) panamensis, g1 (5'-GGTCGGATCTGCATGCATCAT3')-g2 (5' CAAAAAGCGAGGGACTGCGGG-3') for $L$. (V.) guyanensis, m1 (5'-TGCGAGGATAAAGGGAAAGAG-3')-m2 (5'-GTGCCCTGACTTGCATGTCTA-3') for $L$. (L.) mexicana, and a1 (5'-TGCGAGGATAAAGGGAAAGAA-3')a2 (5'-GTGCCCTGACTTGCATGTCTA-3') for $L$. (L.) amazonensis.

The PCR products were separated on $2 \%$ agarose gels containing ethidium bromide.

Statistical analysis. To statistically analyze the association between the zymodemes and cyt b sequences, the Fisher's exact test was used.

\section{RESULTS}

The species assignation of a panel of 17 Argentinean Leishmania stocks and 5 WHO reference strains was performed by $c y t \mathrm{~b}$ gene sequencing and PS-PCR in independent determinations, as is shown in Table 1 .

All these parasites were characterized by MLEE analyzing 12 enzymatic loci. Their enzymatic profiles, phylogenetic relationships, and clinico-epidemiologic characteristics have been reported previously. ${ }^{4}$ Briefly, 14 of the Argentinean Leishmania stocks expressed 3 zymodemes, and termed KMS 1 to KMS 3, were assigned to $L$. (V.) braziliensis. These zymodemes differed from the ones expressed for the WHO reference strains. Two other stocks, grouped in the zymodeme KMS 4, were identified as L. (V.) guyanensis. ${ }^{4}$ The remaining isolate, $\mathrm{MHOM} / \mathrm{AR} / 99 / \mathrm{DMZ}$, also expressed this last zymodeme (data not shown).

Cytochrome $\boldsymbol{b}$ gene sequencing analysis. On the panel of Argentinean Leishmania isolates, the cyt b gene amplification was performed applying PCR with LCBF1 and LCBR2 primers (Figure 1). ${ }^{15}$ The 866 bp DNA fragments obtained for each one of the samples were sequenced and compared with the cyt $\mathrm{b}$ gene sequences of WHO Leishmania reference strains available from DDBJ/EMBL/GeneBank nucleotide sequence databases. Fourteen of these isolates were assigned 
TABLE 1

Species differentiation by three techniques: multilocus enzyme electrophoresis (MLEE) analysis, polymerase chain reaction (PCR) amplification and sequencing of cytochrome $b$ gene (cyt b gene sequencing), and polymorphism-specific PCR (PS-PCR) of 17 Argentinean Leishmania stocks

\begin{tabular}{|c|c|c|c|c|c|c|c|c|c|c|c|}
\hline \multirow[b]{2}{*}{ Stocks designation } & \multirow{2}{*}{$\begin{array}{c}\text { Species } \\
\text { assignation* }\end{array}$} & \multirow[b]{2}{*}{ Zymodeme } & \multirow{2}{*}{$\begin{array}{c}\text { Cyt b } \\
\text { gene } \\
\text { sequence }\end{array}$} & \multicolumn{7}{|c|}{ PS-PCR $\doteqdot$} & \multirow{2}{*}{$\begin{array}{l}\text { Clinical } \\
\text { form }\end{array}$} \\
\hline & & & & $\mathrm{V}$ & $\mathrm{L}$ & $\mathrm{p}$ & $\mathrm{b}$ & $\mathrm{g}$ & $\mathrm{a}$ & $\overline{\mathrm{m}}$ & \\
\hline \multicolumn{12}{|l|}{ Argentinean isolates } \\
\hline MHOM/AR/99/AZ3 & L. (V.) br & KMS 1 & Ab-1 & + & - & - & + & - & - & - & $\mathrm{CL}$ \\
\hline MHOM/AR/02/RLS6 & L. (V.) br & KMS 1 & $\mathrm{Ab}-2$ & + & - & - & + & - & - & - & $\mathrm{CL}$ \\
\hline $\mathrm{MHOM} / \mathrm{AR} / 02 / \mathrm{NSS} 3$ & L. (V.) br & KMS 1 & Ab-1 & + & - & - & + & - & - & - & MCL \\
\hline MHOM/AR/02/MAS5a & L. (V.) br & KMS 1 & Ab-2 & + & - & - & + & - & - & - & $\mathrm{CL}$ \\
\hline MHOM/AR/02/MAS5b & L. (V.) br & KMS 1 & $\mathrm{Ab}-2$ & + & - & - & + & - & - & - & $\mathrm{CL}$ \\
\hline MHOM/AR/03/AAS4 & L. (V.) br & KMS 1 & Ab-1 & + & - & - & + & - & - & - & MCL \\
\hline MHOM/AR/03/OLO1 & L. (V.) br & KMS 1 & Ab-1 & + & - & - & + & - & - & - & $\mathrm{CL}$ \\
\hline MHOM/AR/03/MRO2a & L. (V.) br & KMS 1 & Ab-2 & + & - & - & + & - & - & - & $\mathrm{CL}$ \\
\hline $\mathrm{MHOM} / \mathrm{AR} / 03 / \mathrm{MRO} 2 \mathrm{~b}$ & L. (V.) br & KMS 1 & Ab-2 & + & - & - & + & - & - & - & $\mathrm{CL}$ \\
\hline MHOM/AR/03/HNO3a & L. (V.) br & KMS 2 & $\mathrm{Ab}-2$ & + & - & - & + & - & - & - & $\overline{C L}$ \\
\hline MHOM/AR/03/HNO3b & L. (V.) br & KMS 2 & $\mathrm{Ab}-2$ & + & - & - & + & - & - & - & $\mathrm{CL}$ \\
\hline MHOM/AR/03/FDO4 & L. (V.) br & KMS 2 & Ab-1 & + & - & - & + & - & - & - & $\mathrm{CL}$ \\
\hline MHOM/AR/03/CFO5 & L. (V.) br & KMS 2 & Ab-2 & + & - & - & + & - & - & - & RCL \\
\hline MCAN/AR/02/LPO1 & L. (V.) br & KMS 3 & $\mathrm{Ab}-2$ & + & - & - & + & - & - & - & $\mathrm{CL}$ \\
\hline MHOM/AR/98/LBC1 & L. (V.) gu & KMS 4 & $\mathrm{AB} 095969 \dagger$ & + & - & - & - & + & - & - & $\overline{C L}$ \\
\hline MHOM/AR/99/JDM1 & L. (V.) gu & KMS 4 & AB095969 & + & - & - & - & + & - & - & $\mathrm{CL}$ \\
\hline MHOM/AR/99/DMZ & L. (V.) gu & KMS 4 & AB095969 & + & - & - & - & + & - & - & $\mathrm{CL}$ \\
\hline \multicolumn{12}{|l|}{ Reference strains } \\
\hline MHOM/BR/75/M4147 & L. (V.) gu & KMS 4 & AB095969 & + & - & - & - & + & - & - & $\mathrm{CL}$ \\
\hline MNYC/BZ/62/M379 & L. (L.) me & KMS 5 & AB095963 & - & + & - & - & - & - & + & $\mathrm{CL}$ \\
\hline MHOM/BR/73/M2269 & L. (L.) am & KMS 6 & AB095964 & - & + & - & - & - & + & - & $\mathrm{CL}, \mathrm{DCL}$ \\
\hline MHOM/BR/75/M2904 & L. (V.) br & KMS 7 & AB095966 & + & - & - & + & - & - & - & $\mathrm{CL}$ \\
\hline MHOM/PA/71/LS94 & L. (V.) pa & KMS 8 & AB095968 & + & - & + & - & - & - & - & $\mathrm{CL}$ \\
\hline
\end{tabular}

to $L$. (L.) braziliensis, since the two cyt b gene sequences found, named Ab-1 (5) and Ab-2 (9), showed 99.87\% and $99.75 \%$ similarity to the MHOM/EC/88/INH-03 L. (V.) braziliensis strain sequence (GeneBank accession No. AB095967). The sequences Ab-1 and Ab-2 differed by only one nucleotide base (Figure 2). Statistically, no association between the zymodemes reported and the present sequences was found $(P>1.00)$. A third $c y t \mathrm{~b}$ gene sequence found, and shown for the remaining 3 stocks of the panel, was indistinguishable from the $L$. (V.) guyanensis reference strain MHOM/BR/75/M4147 sequence (GeneBank accession No. AB095969, Table 1).

Leishmania species identification by polymorphismspecific polymerase chain reaction. This analysis is based on the occurrence of either Leishmania subgenus or speciesspecific PCR products corresponding to DNA fragments, which had originally been amplified by arbitrarily primed

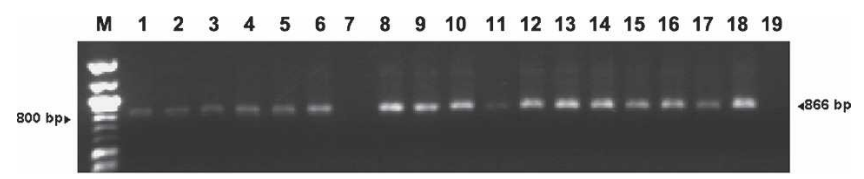

FIGURE 1. Polymerase chain reaction (PCR) products obtained with the primers LCBF1 and LCBR2 for the 17 Argentinean Leishmania isolates. Lane M: size marker (100 bp ladder, BioLabs inc, New England); lane 1, LP01; lane 2, NSS3; lane 3, LBC1; lane 4, DMZ; lane 5, JDM1; lane 6, AZ3; lane 8, OLO1; lane 9, MR02a; lane 10, MR02b; lane 11, HN03; lane 12, HN03; lane 13, FD04; lane 14, CF05; lane 15, MAS5, lane 16, AAS4; lane 17, MAS5; lane 18, RLS6; lane 7 and 19, negative controls. Arrow shows 886 bp (expected amplicon size). For isolates identification see Table 1.
PCR. ${ }^{16}$ First, the samples were simultaneously tested in separate reactions, with Leishmania subgenus-specific primers. All the isolates of the Argentinean panel were identified as $L$. (Viannia) subgenus, since a 168-bp PCR-product with V1-V2 primers, but none with L1-L2 primers were detected. A 78-bp band with the L1-L2 primers was only observed for the WHO reference strains belonging to the L. (Leishmania) subgenus used in this study. In the second step, the species identification was performed using primers b1-b2, p1-p2, and g1-g2 for the samples identified as L. (Viannia) subgenus or a1-a2 and $\mathrm{m} 1-\mathrm{m} 2$ for the strains revealed as L. (Leishmania) subgenus. Thus, a 103-bp PCR-product with the primers b1-b2, but none with the other two pairs of primers was seen in 14 of the Argentinean samples; therefore, they were identified as $L$. $(V$.) braziliensis. The 3 remaining stocks were assigned to $L$. $(V$.$) guyanensis, since only a 79-bp band with the primers$ g1-g2 was seen. (Table 1, Figure 3).

Both, cyt $\mathrm{b}$ gene sequencing and PS-PCR have agreed with MLEE on the Leishmania species identification for each one of the stocks and strains analyzed (Table 1).

\section{DISCUSSION}

The clinico-epidemiologic relevancy of the Leishmania species identification has induced the development and improvement of new molecular tools such as cyt b gene sequencing and PS-PCR, which, as another DNA-based tool, may have many advantages over the classically used methods. ${ }^{12,15,16}$ However, it becomes necessary to test their accuracy on this task comparing with the gold standard technique, a validation assay, as an important previous step in their optimization for 
INH-03/M2904
$\mathrm{Ab}-1$
$\mathrm{Ab}-2$
$\mathrm{M} 4147 / \mathrm{LBC1} / \mathrm{JDM} 1 / \mathrm{DMZ}$

INH-03/M2904

$\mathrm{Ab}-1$

$\mathrm{Ab}-2$

M4147/LBC1/JDM1/DMZ
121 GATAAGAAGTACACATATTTGTTTTACATCATTACTATTTTTTCTTCTTTATGTTCATAT 180

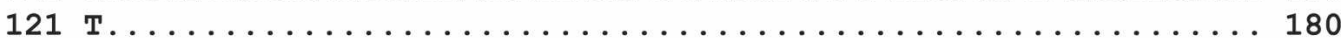

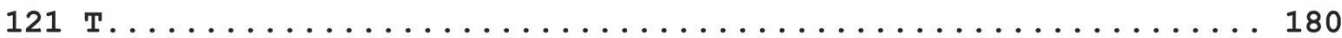

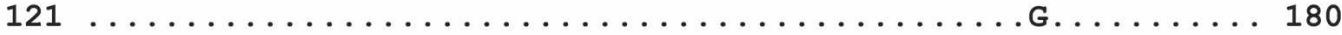

361 TGGTGTTTGATTATGTTATTGAATTGAGGAAGTGAATATATAAATGATTTTACTTTACT 420

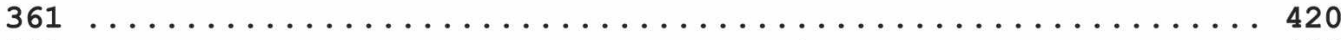

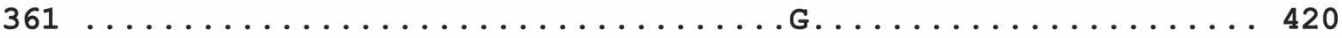

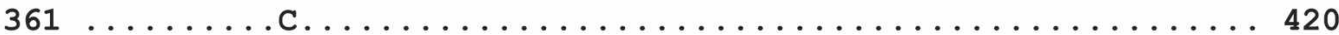

FIGURE 2. Cytochrome $b$ gene alignment. The substitution observed for the Argentinean stocks, Ab-1 and Ab-2 compared with MHOM/ EC/88/INH-03 or HOM/BR/75/M2904 strain fragments sequence (GeneBank no AB095967 and AB095966, respectively) of Leishmania (Viannia) braziliensis are shown. For comparison, the align fragment of the sequence AB095969, corresponding to the L. (V.) guyanensis strains (M4147, LBC1, JDM1, and DMZ) was also included.

applying them in field studies. Besides, the tremendous diversity that exists in the Leishmania genus necessitates the comparison, using local Leishmania isolates from the geographic areas in which the novel techniques are going to be applied. ${ }^{20}$

Under this context, in the current study it was proven that the $c y t \mathrm{~b}$ gene sequencing and PS-PCR methods precisely identified the Leishmania spp. for each one of the local stocks well characterized by MLEE, the gold standard technique, and that they were able to differentiate among the 3 proven species responsible for ATL in Argentina: L. (V.) braziliensis, L. (V.) guyanensis, and L. (L.) amazonensis. ${ }^{4-9}$ In addition, the $c y t \mathrm{~b}$ gene sequences have shown clear interspecific differences, ${ }^{15}$ but high homogeneity among the zymodemes of the two local species analyzed, allowing an accurate species differentiation.

Therefore, these two techniques were validated and could have an application on the species identification of ATL causal agents in this country.

Among the properties of these two molecular methods supporting their applicability in field studies, it should be highlighted that they may not require the isolation and mass cultivation of the parasites, since they can be performed on samples taken directly from the patient (host) lesions and tissues, or from vectors, ${ }^{19,21}$ in contrast with the huge amount of parasites required by MLEE method; the two methods

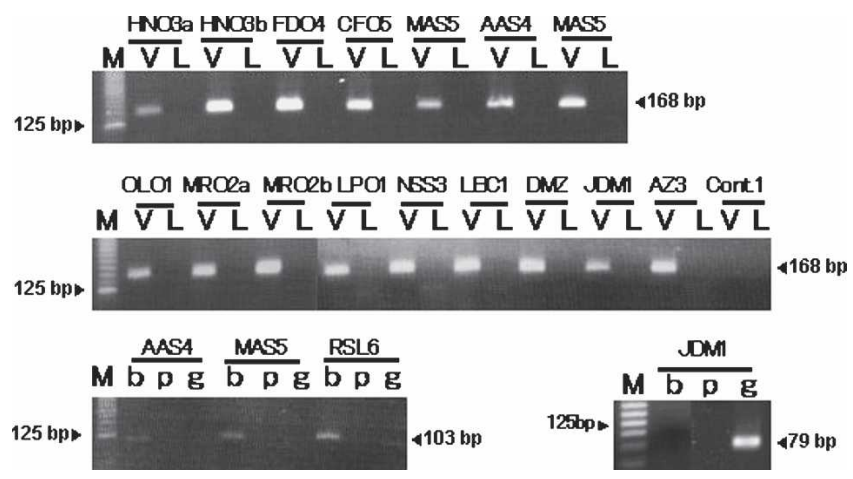

FIGURE 3. Argentinean Leishmania subgenus and species assignation by polymorphism specific (PS)-PCR. Products amplified using V1-V2 (V, expected amplicon size: $168 \mathrm{bp}$ ) and L1-L2 (L, $78 \mathrm{bp}$, no band seen) for Viannia and Leishmania subgenus respectively, b1-b2 (b, 103 bp), p1-p2 (p, 79 bp, no band seen), g1-g2 (g, 79 bp) for $L$. (V.) braziliensis, L. (V.) panamensis and $L$. (V.) guyanensis species, respectively. For isolates identification see Table 1. Lane M: 25 bp DNA Ladder (Gibco BRL). The products were separated on $2 \%$ agarose gels containing ethidium bromide. need relatively shorter times than MLEE for processing; and in the case of PS-PCR, the technique could be applied in laboratories of relatively few resources to analyze great numbers of samples. Nevertheless, the application of the PS-PCR method is only restricted to the differentiation of the 5 major Leishmania spp.- braziliensis, guyanensis, panamensis, amazonensis, and mexicana, responsible for ATL. In the case of the $c y t \mathrm{~b}$ gene sequencing, it could be used for molecular phylogenetic relationship analysis, as a second-line technique in the identification of samples in which the PS-PCR technique may not be conclusive, as in the possible appearance of a Leishmania spp. out of its range, or for the confirmation of very relevant cases. ${ }^{15,19}$

In conclusion, both the cyt $\mathrm{b}$ gene sequencing and the PSPCR techniques showed a total agreement with MLEE, the current gold standard method on the identification of Leishmania spp. on a panel of Argentinean isolates. Hence, this is evidence that their results concerning the present task are very reliable, representing an advantage in the study of the leishmaniases in Argentina, where simple, field-applicable, and reliable diagnostic techniques are extremely necessary.

Received August 10, 2005. Accepted for publication April 20, 2006.

Acknowledgments: We are grateful to Manuel Calvopiña, Hideo Kumazawa, Patricio Diosque, and Daniel Ribble for their contributions and valuable comments. We also thank Kyoko Imamura and the laboratory staffs of the Instituto de Patología Experimental and Instituto de Enfermedades Tropicales for their contributions.

Financial support: This study was supported by the Ministry of Education, Science, Culture and Sports of Japan (Grant Nos. 14256002, 15590371, and 18256004).

Authors' addresses: Jorge D. Marco, Paola A. Barroso, Masataka Korenaga, and Yoshihisa Hashiguchi, Department of Parasitology, Kochi Medical School, Kochi University, Kohasu, Oko, Nankoku, Kochi 783-8505, Japan, Tel-Fax: +81 88880 2415, E-mails: marcojd@med.kochi-ms.ac.jp, paola@med.kochi-u.ac.jp, korenaga@ med.kochi-u.ac.jp, and hasiguti@med.kochi-u.ac.jp. Hiroshi Uezato and Shigeo Nonaka, Department of Dermatology, Faculty of Medicine, University of Ryukyus, 207 Uehara, Nishihara, Okinawa 9030125, Japan, Tel: +81 98895 1152, Fax: +81 98895 1417, E-mails: huezato@med.u-ryukyu.ac.jp, and shigeo@med.u-ryukyu.ac.jp. Tatsuyuki Mimori, Department of Tumor Genetics \& Biology, Graduate School of Medical Science, Kumamoto University, 1-1-1 Honjo, Kumamoto 860-8556, Japan, Telephone: +81 96373 5117, Fax: +81 96 373 5120, E-mail: tmimori@kaiju.medic.kumamoto-u.ac.jp. Miguel A. Basombrío, Instituto de Patología Experimental, Facultad de Ciencias de la Salud, Universidad Nacional de Salta, Buenos Aires 177, (4400) Salta, Argentina, Tel-Fax: +54 387 4255333, E-mail: basombri@unsa.edu.ar. Néstor J. Taranto, Instituto de Enfermedades Tropicales, Sede Regional Orán, Universidad Nacional de Salta, Al- 
varado 751, San Ramón de la Nueva Orán, (4530) Salta, Argentina, Tel-Fax: +54 3878 421924, E-mail: inst_trop@arnet.com.ar.

Reprint requests: Jorge D. Marco, Department of Parasitology, Kochi Medical School, Kochi University, Kohasu, Oko, Nankoku, Kochi 783-8505, Japan. Tel-Fax: +81 88880 2415. E-mail: marcojd@ med.kochi-ms.ac.jp.

\section{REFERENCES}

1. Dedet JP, 2002. Current status of epidemiology of leishmaniases. Farrell JP (ed). Leishmania, World-class Parasites: Volume 4. Boston, MA: Kluwer Academic Publishers, 1-10.

2. Castilho TM, Shaw JJ, Floeter-Winter LM, 2003. New PCR assay using glucose-6-phosphate dehydrogenase for identification of Leishmania species. J Clin Microbiol 41: 540-546.

3. Navin TR, Arana BA, Arana FE, Bermen JD, Cajón JF, 1992. Placebo-controlled clinical trial of sodium stibogluconate (Pentostam) versus ketaconazole for treating cutaneous leishmaniasis in Guatemala. $J$ Infect Dis 165: 528-534.

4. Marco JD, Barroso PA, Calvopiña M, Kumazawa H, Furuya M, Korenaga M, Cajal SP, Mora MC, Rea MMJ, Borda CE, Basombrío MA, Taranto NJ, Hashiguchi Y, 2005. Species assignation of Leishmania from humans and canine American tegumentary leishmaniasis cases by multilocus enzyme electrophoresis in north Argentina. Am J Trop Med Hyg 72: 606-611.

5. Shaw JJ, 2002. New World leishmaniasis: the ecology of leishmaniasis and the diversity of leishmanial species in Central and South America. Farrell JP (ed). Leishmania, World-class Parasites: Volume 4. Boston, MA: Kluwer Academic Publishers, $11-31$.

6. Salomón OD, Sosa Estani S, Rossi GC, Spinelli GR, 2001. Presencia de Lutzomya longipalpis y situación de la leishmaniosis visceral en Argentina. Medicina (B Aires) 61:174-178.

7. Nocito I, Serra E, Montero A, 2002. Visceral involvement due to Leishmania mexicana in a patient with acquired immunodeficiency syndrome. Am J Med 113: 260-262.

8. Martín-Sánchez J, Navarro-Mari JM, Pasquau-Liano J, Salomón OD, Morillas-Marquez F, 2004. Visceral leishmaniasis caused by Leishmania infantum in a Spanish patient in Argentina: What is the origin of the infection? Case report. BMC Infect Dis 4: 20.

9. Córdoba Lanús E, Pinero JE, González AC, Valladares B, de Grosso ML, Salomón OD, 2005. Detection of Leishmania braziliensis in human paraffin-embedded tissues from Tucumán, Argentina by polymerase chain reaction. Mem Inst Oswaldo Cruz 100: 187-192.

10. Romero GA, Guerra MV, Paes MG, Macedo VO, 2001. Comparison of cutaneous leishmaniasis due to Leishmania (Viannia) braziliensis and $L$. $(V$.) guyanensis in Brazil: clinical findings and diagnostic approach. Clin Infect Dis 32: 1304-1312.
11. Romero GA, Guerra MV, Paes MG, Macedo VO, 2001. Comparison of cutaneous leishmaniasis due to Leishmania (Viannia) braziliensis and $L$. (V.) guyanensis in Brazil: therapeutic response to meglumine antimoniate. Am J Trop Med Hyg 65: 456-465.

12. Marfurt J, Nasereddin A, Niederwieser I, Jaffe CL, Beck HP, Felger I, 2003. Identification and differentiation of Leishmania species in clinical samples by PCR amplification of the miniexon sequence and subsequent restriction fragment length polymorphism analysis. J Clin Microbiol 41: 3147-3153.

13. Victoir K, De Doncker S, Cabrera L, Álvarez E, Arévalo J, Llanos-Cuentas A, Le Ray D, Dujardin JC, 2003. Direct identification of Leishmania species in biopsies from patients with American tegumentary leishmaniasis. Trans $R$ Soc Trop Med Hyg 97: 80-87.

14. Guizani I, 2004. Molecular tools for studying the epidemiology of leishmaniasis. World Health Organization, eds. Reports of the Scientific Working Group on Leishmaniasis. Geneva: WHO, $54-70$.

15. Luyo-Acero G, Uezato H, Oshiro M, Takei K, Kariya K, Katakura K, Gómez EAL, Hashiguchi Y, Nonaka S, 2003. Sequence variation of the cytochrome $b$ gene of various human infecting members of the genus Leishmania and their phylogeny. Parasitology 128: 483-491.

16. Mimori T, Sasaki J, Nakata M, Gomez EAL, Uezato H, Nonaka S, Hashiguchi Y, Furuya M, Saya H, 1998. Rapid identification of Leishmania species from formalin-fixed biopsy samples by polymorphism-specific polymerase chain reaction. Gene 210 $179-186$.

17. World Health Organization, 2004. Report of the Scientific Working Group on Leishmaniasis. Geneva: WHO, 5-6.

18. Diosque P, Barnabé C, Padilla AM, Marco JD, Cardozo RM, Cimino RO, Nasser JR, Tibayrenc M, Basombrío MA, 2003. Multilocus enzyme electrophoresis analysis of Trypanosoma cruzi isolates from a geographically restricted endemic area for Chagas disease in Argentina. Int J Parasitol 33: 997-1003.

19. Kato H, Uezato H, Katakura K, Calvopina M, Marco JD, Barroso PA, Gomez EAL, Mimori T, Korenaga M, Iwata $\mathrm{H}$, Nonaka S, Hashiguchi Y, 2005. Detection and identification of Leishmania species within naturally infected sand flies in the Andean areas of Ecuador by a polymerase chain reaction. $\mathrm{Am}$ J Trop Med Hyg 72: 87-93.

20. Cupolillo E, Grimaldi G Jr, Momen H, Beverley SM, 1995. Intergenic region typing (IRT): A rapid molecular approach to the characterization and evolution of Leishmania. Mol Biochem Parasitol 73: 145-155.

21. Mimori T, Matsumoto T, Calvopiña M, Gomez EA, Saya H, Katakura K, Nonaka S, Shamsuzzaman SM, Hashiguchi Y, 2002. Usefulness of sampling with cotton swab for PCRdiagnosis of cutaneous leishmaniasis in the New World. Acta Trop 81: 197-202. 